\title{
Tópicos en la regulación de Internet: neutralidad de red y administración de tráfico
}

Revista Latinoamericana de Economía y Sociedad Digital

Issue 2, agosto 2021

\section{Autores: Nestor Bruno}

DOI: $10.53857 /$ ECPI9934

Publicado: 25 agosto, 2021

Recibido: 10 marzo, 2021

Cita sugerida: Bruno, Néstor (2021) "Tópicos en la regulación de Internet: neutralidad de red y administración de tráfico" en Revista Latinoamericana de Economía y Sociedad Digital, Issue 2

Licencia: Creative Commons Atribución-NoComercial 4.0 Internacional (CC BY-NC 4.0)

Tipo: Ensayo

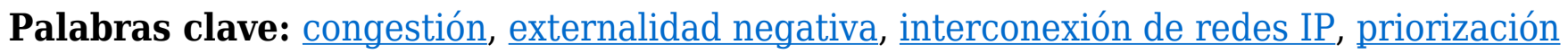

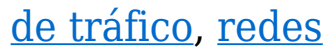

\section{Resumen}

Una práctica de transmisión de datos en internet que se desvíe del precepto general conocido como "mejor esfuerzo" es calificada como una violación al principio de neutralidad de red. Presentamos un modelo no neutral de contrato entre proveedores de acceso a internet (ISP) y proveedores de contenido (CAP) en base al concepto de tráfico priorizado. Evaluamos sus beneficios comparándolo con una situación neutral y hallamos que en la modalidad no neutral se reduce el cargo. La modelización de la congestión de red en conjunto con la determinación de los beneficios de ISP y CAP y la consideración de una forma específica de trafico administrado genera resultados que difieren de los argumentos regulatorios simples[1] donde siempre es beneficioso para los ISP apartarse del régimen de neutralidad de red y extraer parte de los recursos de los proveedores de contenido (CAP)[2] a pesar que aun en éste caso el excedente total del mercado puede aumentar. 


\section{Abstract}

A practice of data transmission in the internet that is diverted from the general precept known as "best effort" is qualified as a violation of the neutrality principle of the network. We present a non-neutral model of contract between internet service providers (ISP) and content providers (CAP) based on the concept of prioritized traffic. We have assessed the benefits comparing them with a neutral situation and we have discovered that the nonneutral modality reduces the charge. The modelling of the network congestion together with the determination of the benefits of ISP and CAP and the consideration of a specific way of administrative traffic produces results that are different from the simple regulatory arguments where it is always beneficial for the ISP not to follow the network neutrality regime and extract part of the resources of the content providers (CAP) although, even in this case, the total surplus of the market can increase.

\section{Resumo}

Uma prática de transmissão de dados na internet que se desvia do preceito geral conhecido como "melhor esforço" é qualificada como uma violação do princípio de neutralidade de rede. Apresentamos um modelo não neutro de contrato entre provedores de acesso à internet (ISPs) e provedores de conteúdo (CAP) com base no conceito de priorização de tráfego. Avaliamos os seus benefícios comparando-o com uma situação neutra e descobrimos que na modalidade não neutra a carga é reduzida. A modelização do congestionamento de rede em conjunto com a determinação dos benefícios de ISP e CAP e a consideração de uma forma específica de tráfego administrado gera resultados que diferem dos argumentos regulatórios simples, onde é sempre benéfico para os ISPs se afastarem do regime de neutralidade de rede e extrair parte dos recursos dos provedores de conteúdo (CAP), apesar do fato de que, mesmo neste caso, o excedente total do mercado pode aumentar.

\section{Introducción}

Una definición aceptada de neutralidad de la red indica que todo el tráfico que circula por una red debe ser tratado de igual forma, independientemente del contenido, la aplicación, el servicio, el dispositivo o la dirección del que lo envía o lo recibe (BEREC, 2012), conforme al principio denominado "principio del mejor esfuerzo". Cualquier práctica que se desvíe del principio enunciado resulta en una violación de la neutralidad de la red.

En la actualidad el tráfico cursado por las redes aumenta rápidamente (Cisco, 2020) y las aplicaciones son más sensibles al retardo, por lo que el valor que los consumidores derivan de las mismas se encuentra comprometido. Ejemplo de las mismas son juegos en línea, 
servicios de video de realidad aumentada, telemedicina, etcétera.

La presencia de riesgos de congestión, junto a consumidores heterogéneos en su demanda de acceso a internet y a proveedores de aplicaciones y de contenido que demandan acceso de calidad mientras son restringidos por el principio de mejor esfuerzo resulta en una situación ineficiente tanto estática y como dinámicamente. Aplicar medidas de priorización de tráfico redundaría en un aumento del bienestar de los consumidores en la forma de una reducción de los abonos pagados por acceder a internet, tal como se demostrará en el presente análisis, y permitirá generar fondos que expandan la capacidad de los ISP para adaptar los servicios de acceso a las demandas de los consumidores y reaccionar ante factores exógenos.

Para evaluar la validez de las afirmaciones presentamos un modelo de demanda de conexión a Internet basado en la teoría de las colas, toda vez que el ancho de banda y la congestión debida al aumento sustancial del tráfico son elementos centrales del debate. Modelamos también las interrelaciones entre un operador de red (ISP), dos proveedores de contenido (CAP) y usuarios heterogéneos con respecto a sus preferencias por los servicios de contenido a la Hotelling. Ofrecemos un análisis económico formal sobre los efectos de la reglamentación de la neutralidad de la red en los beneficios tanto de los ISP como de los CAP.

Comparamos el equilibrio del mercado en el que se permite al ISP proporcionar un servicio de dos niveles donde subasta el servicio de "vía rápida" para solo un CAP con el equilibrio de mercado cuando el ISP no puede discriminar la velocidad de entrega del contenido.

Esta comparación de equilibrios de corto plazo arroja dos conclusiones importantes. En primer lugar, en una red no neutral ambos proveedores de contenido pueden participar en un juego del tipo "dilema del prisionero" para recibir la primera prioridad en la entrega de contenido y no estar en peor situación. La decisión de los ISP de preferir el régimen discriminatorio a la red neutral depende del equilibrio entre el cargo de acceso a la red cobrado a los usuarios finales y los ingresos provenientes de los CAP por el comercio de la primera prioridad. En segundo lugar, encontramos que, bajo los supuestos presentados, el cargo de acceso cobrado a los usuarios finales disminuye en el escenario de no neutralidad.

En la Sección I presentamos el concepto de neutralidad, la conformación actual del ecosistema de Internet y su cadena de valor, los pagos involucrados y los elementos salientes para el análisis. En la Sección II presentamos la bibliografía relacionada, en la Sección III presentamos un modelo de no neutralidad (discriminación) ${ }^{[1]}$ entre los ISP y los CAP en base a concepto de "tráfico administrado" para acceso a Internet y evaluamos las diferencias en términos de beneficios para el ISP (y los CAP) entre una situación de neutralidad y otra de no neutralidad; finalmente en la Sección IV presentamos las conclusiones y comentarios. 


\section{Neutralidad de red, ecosistema de internet y su cadena de valor}

Mencionamos en la introducción que, bajo la definición usual de neutralidad de red, todos los paquetes que atraviesan internet son tratados de manera igualitaria, principio éste conocido también como del "mejor esfuerzo". Cualquier práctica que se desvíe resulta en una violación de este principio.

Este debate se ha visto exacerbado por el hecho que en los últimos años el volumen de tráfico de internet ha crecido exponencialmente (Cisco, 2020 y IEA, 2019), obligando a los ISP a actualizar continuamente la capacidad de su red. Dos de los principales ISP de Estados Unidos fueron los primeros casos donde se planteó explícitamente el reemplazo del principio de neutralidad de red. ${ }^{[2]}$

Los partidarios de la neutralidad de la red sostienen que, dado que Internet ha sido neutral desde su creación, debe mantenerse libre y abierto a todos. Argumentan que una desviación del régimen de neutralidad reduciría la innovación en servicios de internet (entendida como una mayor afluencia de CAP), y sostienen también que la inversión de los ISP no se vería afectada en un régimen de neutralidad toda vez que los ISP seguirán invirtiendo en capacidades de banda ancha ya que es la única manera de mantener su demanda. Por último, argumentan que los usuarios finales consideran tanto el valor de las cuotas de suscripción que pagan a los ISP como la variedad del contenido al que acceden mediante internet y la calidad de su conexión (Crocioni, 2011 y Cheng et al., 2009). Los partidarios de la neutralidad de la red abarcan sobre todo a grandes empresas de contenidos en internet.

Por el otro lado, y a modo de ejemplo de la intensidad del debate sobre neutralidad de red, los ISP se oponen a las regulaciones y afirman que desalientan la inversión en redes de banda ancha. La lógica es que no tendrían ningún incentivo invertir en capacidad de red a menos que los proveedores de contenido, que son quienes determinan el uso intensivo de las aplicaciones multimedia de banda ancha, paguen una prima por la cantidad de contendidos que agregan a la red y que determina que el tráfico llegue a mayores niveles de congestión.

La inversión en los activos fijos que compone una red de acceso a Internet, su propiedad, la disponibilidad y el impacto competitivo en el mercado, han generado un sustancial debate regulatorio. Tradicionalmente se exigió intervención regulatoria en presencia de fallas de mercado. Una de estas fallas se verifica cuando un grupo de firmas compiten entre sí en la provisión de un bien y una de ellas tiene la propiedad monopólica de un insumo o infraestructura que es indispensable para la oferta de dicho bien. Surge entonces el problema de cómo preservar y aumentar la competencia en estos mercados. La denominada doctrina de las facilidades esenciales ${ }^{[3]}$ fue el abordaje analítico para estos problemas competitivos y para una serie de soluciones transitorias regulatorias. 
Si bien mercado de Internet se ha desarrollado en un entorno desregulado, las medidas implementadas bajo el paradigma de las facilidades esenciales y los aspectos controvertidos que emergieron guardan similitudes destacables respecto de la necesidad de acordar contratos con precios que informen la escasez relativa de la capacidad de las redes, toda vez que internet es una red de redes.

Formalmente la Ley de Telecomunicaciones de 1996 (U. S. Congress, 1996) en los Estados Unidos buscó implementar esta doctrina a través de lo que se conoce como desagregación del bucle de acceso local ${ }^{[4]}$. Los reguladores nacionales de Europa (Communication from the Commission, 2000) implementaron también el mismo procedimiento. En la Argentina existen también antecedentes al respecto. Los más importantes son sin duda el Decreto 764/2000 y la reciente Resolución 286/2018 del Ministerio de Modernización (que aprobó el denominado Reglamento Nacional de Interconexión).

El proceso de apertura de redes establecido por FCC generó inicialmente una rápida afluencia de competidores al mercado y que fragmentaron su estructura, pero en forma no sustentable: provocó una alta rotación de empresas que entraron al mercado buscando una rentabilidad garantizada por el regulador que rápidamente se erosionó y, al mismo tiempo, faltaron incentivos para que los operadores establecidos realizaran inversiones, ya que enfrentaban precios minoristas con severas tendencias a la baja y valores mayoristas remunerados en base a costos incrementales de largo plazo, significativamente distintos a los costos históricos promedios de los equipos existentes ${ }^{[5]}$.

En el caso americano, la complejidad normativa introducida resultó además en largos procedimientos de determinación de costos mayoristas y motivó altos costos judiciales regulatorios, por lo que la FCC finalmente eliminó en 2003 la posibilidad de solicitar estos servicios mayoristas por parte de los proveedores de acceso a Internet (ISP) para dar solo el servicio de acceso a Internet residencial (FCC News, 2003; FCC, 2005).

\section{I.1 Ecosistema de Internet y su cadena de valor}

Actualmente existen cadenas verticales complejas y acuerdos comerciales denominados ecosistemas que, por un lado, permiten a los consumidores acceder a los contenidos y aplicaciones a través de las redes de Internet, y por el otro, permiten a los proveedores de contenido llegar a los consumidores. Para conectarse a Internet, los consumidores se suscriben a un (ISP) ${ }^{[6]}$. Los operadores de redes móviles, junto con los operadores de redes fijas (incluye a los operadores de cable) y los denominados operadores virtuales ${ }^{[7]}$ revisten la calidad de ISP.

Las redes de mayor nivel constituyen la columna vertebral de Internet y tienen conexiones directas entre sí basadas en el concepto de "tráfico entre pares" ${ }^{[8]}$, mientras que las redes de jerarquía menor compran "tránsito" a otras redes de mayor infraestructura para acceder a Internet y ofrecen sus servicios de acceso a Internet a los clientes finales. El intercambio de tráfico entre redes se denomina punto de intercambio de Internet (IXP) y es una 
infraestructura física a través de la cual los ISP intercambian el tráfico de Internet entre sus redes.

Por otro lado, los CAP como Google, YouTube o Facebook necesitan subir su contenido (o aplicaciones) a internet a fin de que estén visibles y disponibles para los consumidores finales. Celebran contratos de servicios de almacenamiento de datos ${ }^{[9]}$ (webhosting) para su contenido. Actores significativos, con infraestructura propia, son también las denominadas "redes de distribución de contenido" (Content Distribution Networks - CDN). Estos utilizan su propia red de servidores en el borde de la red para almacenar información y contenido cerca de los consumidores. En otras palabras, los consumidores pueden acceder al contenido a través de servidores mucho más cercanos, sin necesidad que el tráfico atraviese el core de Internet.

Debido al aumento del riesgo de congestión, algunos CAP como Yahoo y Google han creado su propios CDN, mientras que otros utilizan los servicios prestados por terceros, como Akamai. Si bien los consumidores no notan diferencia en la calidad de su conexión a Internet, los $\mathrm{CDN}$ son un intento de reducir los riesgos de congestión con los que se topa un modelo de mejor esfuerzo, que tal como se ha expresado, es el modelo establecido en Internet.

Los CAP pagan entonces a participantes diferentes al ISP por subir su contenido, por alojamiento web y por el uso de la capacidad a los CDN. Reciben además usualmente ingresos indirectos por publicidad. Resulta conveniente destacar que todos estos actores mencionados prestan servicios diferentes a los ISP. Por su parte los consumidores pagan a los proveedores de servicios de acceso Internet (ISP) por la conectividad y así poder acceder a los contenidos deseen consumir de y enviar hacia la red.

\section{I.2 Elementos salientes de la cadena de valor presentada}

Dos cuestiones centrales ocupan un lugar preponderante en cadena de valor presentada: en primer lugar, los CAP no realizan pagos a los ISP a los que se suscriben los consumidores para acceder a Internet. Los CAP pagan a los distintos participantes de la cadena de valor para llevar y almacenar su contenido cerca del ISP elegido por el consumidor, pero no para la última parte del viaje a través de la red del ISP, que es la que mayor inversión y capilaridad de red requiere. Este es uno de los aspectos críticos actualmente en debate regulatorio: al no existir precios desde los ISP a los CAP que informen la escasez relativa de la capacidad de redes, se genera un desequilibrio de incentivos: el contenido subido por los CAP crece a tasa exponenciales, fuertemente desalineadas de los programas de inversión de los ISP, y provocando congestión a todos los consumidores que experimentan una menor calidad de servicio. Esta última consecuencia es usualmente reclamada al ISP, que es como se ha señalado, con quien contrata el consumidor final el acceso a Internet. Recientemente, prácticas comerciales conocidos como esquemas de zero rate se han masificado: mediante estas un operador móvil no toma en cuenta el volumen de datos consumido por una aplicación en particular, un contenido o servicios dentro del volumen de datos que el cliente 
contrata. El motivo de esta práctica consiste en bonificar consumos que son atractivos los clientes y que son compensadas con mayores usos dentro de un determinado plan o acceso a planes más onerosos que incluyen la modalidad del zero rate $e^{[10]}$.

El segundo aspecto del debate es que el contenido y las aplicaciones viajan en gran medida según la modalidad descripta como de mejor esfuerzo, lo que implica sin garantía de calidad. Esta es la razón por la cual el contenido a veces tarda en cargarse en los equipos de los consumidores finales (PC, tablet o celular), en las demoras en descargar archivos del correo electrónico o imágenes de video que vemos congeladas. Si bien esto es suficiente para la mayoría de los contenidos y aplicaciones que no son sensibles a retrasos, podrían sin embargo impactar negativamente en el valor de las aplicaciones para las cuales los retrasos (latencia) resulta crítica. Este es el caso de juegos en línea, video, aplicaciones de realidad aumentada y telemedicina ${ }^{[11]}$, entre otras.

A pesar de que la mayor parte del tráfico circula bajo el modelo de mejor esfuerzo, varios ISP actualmente participan en alguna forma de gestión de tráfico, priorizando un tipo de tráfico sobre otro (Crocioni, 2011). Los consumidores generalmente pagan una tarifa plana para obtener conexión a Internet. Sin embargo, el tráfico podría cobrarse en forma más eficiente mediante precios relacionados con el uso ${ }^{[12]}$. Si bien esto es posible, los consumidores no las prefieren, ya que históricamente se han alejado de mecanismo de precios que incluyan acceso medido. Diseñar tarifas para el uso de aplicaciones específicas resulta ser menos eficiente que proponer pagos por priorización de tráfico a los $\mathrm{CAP}^{[13]}$.

\section{Bibliografía Relacionada}

Las contribuciones iniciales corresponden al campo de las telecomunicaciones. Hermalin y Katz (2007) determinan que una obligación de no discriminación (neutralidad de red) obligaría a los ISP a ofrecer un servicio de acceso con calidad intermedia, y al que solo se conectarían los consumidores que valoraran el acceso a internet de esa calidad. Solo algunos consumidores suscriben contrato y participan, mejorando su bienestar.

El efecto global de una obligación de no discriminación es negativo en términos de bienestar. Por último, en presencia de una gran proporción de costos fijos y comunes en la provisión de acceso a Internet, la discriminación de precios es un método eficiente de recuperar dichos costos sobre la base de las elasticidades de precios de demanda relativas dentro y en los dos lados de este mercado. En esta misma línea, Economides y Hermalin (2012) demuestran que la capacidad de discriminación de precios aumenta los incentivos para invertir, creando un equilibrio entre las eficiencias estáticas y dinámicas.

Varias contribuciones recientes discuten la selección de contenido sensible a la congestión por medio de precios y niveles de calidad diferenciados, un aspecto clave del debate de neutralidad de la red y que en el presente trabajo incluimos explícitamente. Ejemplo de esta línea de análisis es Krämer y Wiewiorra (2012), donde estudian alternativas a la neutralidad 
de red mediante la implementación de un régimen de niveles de calidad de servicio, en el que un proveedor de servicios de internet cobra por la priorización sobre una base no discriminatoria. Encuentran que la calidad de la organización en niveles de servicio puede ser más eficiente a corto plazo porque asigna mejor la capacidad de red existente y a largo plazo porque proporciona mayores incentivos a la inversión debido a la creciente demanda de servicios prioritarios por la entrada de nuevos proveedores de contenido sensible a la congestión. El régimen de red más eficiente depende de la distribución de la sensibilidad a la congestión entre los proveedores de contenido, pero una directriz es que el régimen que proporciona mayores incentivos para las inversiones en infraestructura es más eficiente a largo plazo.

En esta misma línea podemos mencionar las contribuciones de Choi et al. (2015), Bourreau et al. (2015) y Reggiani y Valletti (2016). Resulta relevante también el análisis de Kourandi y Valletti (2015) donde proponen un modelo bilateral, con dos plataformas competidoras y un continuo de proveedores de contenido (CAP). Estudian el efecto de una regulación de neutralidad en las inversiones de capacidad en el mercado del acceso a Internet y en la innovación en el mercado de contenidos. En virtud del régimen discriminatorio (no neutral) las plataformas cobran una tasa prioritaria a los CAP que están dispuestos a entregar su contenido en una vía rápida. Constatan que, en virtud de la discriminación, las inversiones en capacidad de banda ancha y la innovación de contenidos son superiores a las de la neutralidad de la red. El bienestar total, entonces, aumenta.

Greensten et al. (2016) realizan una descripción de las modalidades contractuales más comunes y pagos generados entre los participantes del mercado de acceso a Internet. Respecto al impacto de esquemas de precios únicos versus precios diferenciados, consideran un modelo en la que dos proveedores de contenido idénticos enfrentan la demanda por contenido de usuarios que difieren en su disposición a pagar una cuota de suscripción. Esto resulta en una demanda elástica de acceso. Y sólo algunos usuarios pueden estar dispuestos a suscribirse si el precio es alto. Cuando un ISP decide cobrar a un proveedor de contenido por el tráfico (priorizando la velocidad) que genera, determinan que la tarifa de suscripción pagada por los usuarios finales disminuirá si los proveedores se encuentran en un entorno competitivo, ya que sus ganancias, de todas las fuentes, se mantienen a un nivel normal por el propio proceso competitivo.

Jullien y Sand-Zantman (2018) exploran la determinación de precios óptimos de acceso a la Internet con proveedores de contenido diferenciados. Dado que estos últimos no pueden afectar directamente el comportamiento del consumidor a través del precio de su contenido, implementan el mecanismo conocido como zero-rate. Aumenta así el consumo de contenido de alto valor y reduce los incentivos de las redes para excluir el contenido de bajo valor. El efecto de bienestar de permitir esta discriminación de precios depende de la proporción de contenido dirigido y del valor de los contenidos. El análisis se extiende en varias direcciones (precios unilaterales, red competidora, costo heterogéneo, contenido de pago). 


\section{II.1 Comentarios salientes sobre la bibliografía relacionada}

Los trabajos enumerados presentan las cadenas verticales complejas a través de las cuales los contenidos llegan a los consumidores, y explicitan los varios trade off involucrados. Reportan análisis donde una obligación de no discriminación resulta negativa en términos de bienestar, y encuentran que la presencia de diferentes niveles de servicio puede ser más eficiente a corto plazo porque asigna mejor la capacidad de red existente y a largo plazo porque proporciona mayores incentivos a la inversión debido a la creciente demanda de servicios prioritarios por la entrada de nuevos proveedores de contenido sensible a la congestión. En esta línea analizaremos los impactos de la administración del tráfico a Internet mediante la oferta del ISP de dos niveles de servicios.

Presentaremos un modelo basado en la teoría de las colas para modelar la congestión en la red. El mismo es un marco estándar ampliamente utilizado en la investigación de operaciones para estudiar los problemas de congestión y la fijación de precios prioritarios. La posible necesidad de racionamiento en el acceso a Internet se encuentra en las causas fundamentales del debate. Modelamos un operador de red (ISP) y dos proveedores de aplicaciones (CAP).

Modelamos la demanda de acceso a Internet y de contendidos, los beneficios de los ISPs y CAPs, y comparamos el equilibrio del mercado en el que se permite al ISP proporcionar un servicio de dos niveles y vender la "vía rápida" a un solo proveedor de contenido, con el equilibrio en el que no puede discriminar la velocidad de entrega del contenido. Hallamos que en la red no neutral se reduce el cargo de acceso que el ISP puede cobrar a los usuarios finales. La decisión de los ISP de preferir el régimen discriminatorio a la red neutral depende del equilibrio entre el cargo de acceso a la red cobrado a los usuarios finales -menor que en el escenario de no neutralidad-y los ingresos provenientes de los CAP por el comercio de la primera prioridad.

\section{Modelo de tráfico administrado para acceso a internet}

Nuestro análisis se centra en un mercado en el que los ISP proporcionan el servicio de acceso a Internet a consumidores finales para acceder a los contenidos generados por los $\mathrm{CAP}$, al tiempo que modelamos la congestión y la posibilidad de proponer un cargo diferencial por priorizar la entrega del contenido en ausencia de la regulación de neutralidad de la red.

\section{III.1 Descripción del modelo básico de acceso a Internet}

El ISP ofrece acceso a Internet a través de su infraestructura de red a los usuarios finales para acceder a los contendidos generados por dos CAP. A los clientes finales el ISP les cobra un precio $\boldsymbol{p}$. En el marco de la neutralidad de la red el ISP no cobra a los CAP por 
enviar información a través de su red a los usuarios finales, ni discrimina entre los CAP la velocidad de entrega de sus contenidos. Conforme lo mencionado los ISP que proporcionan servicio de alojamiento a los CAP son diferentes del ISP locales que proporcionan acceso a los usuarios finales. Bajo la premisa de neutralidad de red los CAP pagan tarifas al operador de red de alojamiento solo una vez en el origen, y no se les exige que paguen adicionalmente por el tránsito en la red de cada ISP. Los ISP locales que proporcionan el acceso de última milla a los consumidores finales no pueden exigir una compensación adicional a los CAP. Sin la regulación de la neutralidad de la red, en cambio el ISP puede vender la primera prioridad -el derecho a ser atendido antes- a cualquiera de los dos proveedores de contenido.

\section{2 Mercado minorista, los consumidores y los proveedores de contenido}

Los consumidores son heterogéneos con respecto a sus preferencias por los servicios de contenido, y son modelados a la Hotelling, con una masa normalizada a uno. Los $\mathrm{CAP}_{1} \mathrm{y}$ $\mathrm{CAP}_{2}$ se encuentran en los extremos izquierdo y derecho respectivamente de un segmento lineal, con longitud también normalizada a uno. Un consumidor situado en " $\chi$ " paga el costo de transporte de "tx" y "t-tx" para consumir los servicios de $\mathrm{CAP}_{1}$ y CAP ${ }_{2}$ respectivamente.

El costo de transporte por unidad de distancia, " $t$ ", representa el grado de diferenciación del producto. Asumimos que cada consumidor posee funciones de demanda establecidas sobre el contenido de uno de los dos CAP. Al igual que en Mendelson (1985) asumimos que la tasa de demanda de contenido de cada consumidor sigue un proceso de Poisson con un cociente de riesgo $\varphi^{[14]}$ que representa la intensidad de su demanda de contenido. La capacidad de la red se denota como $\xi$.

El tiempo de servicio tomado para entregar el contenido de los CAP a los usuarios finales es distribuido exponencialmente con media de $1 /$ ६: mayor capacidad de red implica un tiempo de servicio más corto. Asumimos que en el corto plazo la capacidad $\xi$ es fija. Cada consumidor esta diferenciado por una tasa de solicitud de contenido $\varphi$, y deriva una utilidad bruta de $u(\varphi)=v$ sobre cualquiera de los servicios de contenido, y además $v$ es lo suficientemente grande como para que el mercado esté completamente cubierto.

Consideraremos que cada proveedor de contenido obtiene un flujo de ingresos " $r_{c}$ " proveniente del contenido que genera ${ }^{[15]} \mathrm{y}$ los consumidores demandan conforme a su tasa de solicitud de contenido $\varphi$. La asimetría en " $r_{c}$ " refleja las diferencias en las capacidades de los CAP para satisfacer las demandas de contenido de sus consumidores. El costo de servir la solicitud de cada consumidor está dado por " $C_{c}$ ". El beneficio del CAP $\mathrm{C}_{\mathrm{i}}$ viene dado por $b_{i=}$ $y_{c i}-c_{c i}$ donde $b_{1} \geq b_{2} \geq 0$

Las fuentes de asimetría en los beneficios de los CAP pueden generarse tanto a nivel de los ingresos, de los costos o en una combinación de ambos ${ }^{[16]}$.

\section{3 Congestión de redes, un modelo usual}


Para modelar la congestión en la red, adoptamos el marco estándar del sistema de colas que ha sido ampliamente utilizado en la investigación de operaciones para problemas de congestión y fijación de precios prioritarios (Naor, 1969, Balachandran, 1972, Edelson et al., 1975 y Meldenson et al., 1990). Las razones de esta elección del modelo obedecen por una parte a que las causas fundamentales del debate sobre la neutralidad de la red son la escasez de ancho de banda y la necesidad de racionamiento debido a los aumentos sustanciales de la demanda de acceso a internet.

Y además esta configuración constituye una muy buena aproximación para el proceso de llegada a los sistemas reales, en los que el número de clientes es lo suficientemente grande como para que el impacto de un solo cliente en el rendimiento del sistema sea muy pequeño, y todas las decisiones de los clientes de utilizar el sistema son independientes de las de otros usuarios. Estos micro fundamentos poseen además propiedades útiles con las que modelar la congestión sin ningún tipo de suposición ad hoc.

Cada consumidor tiene, entonces, un tiempo de espera dado por:

$$
w=(\xi-\varphi)^{-1}
$$

Donde $\varphi$ denota la tasa bruta de solicitud de contenido en la red (con la normalización de la masa de consumidores a uno), y con capacidad de la red $\xi>\varphi$. El tiempo de espera aumenta en $\varphi$, y disminuye en $\xi$. Si normalizamos el coste de retraso por unidad de tiempo a uno, entonces el tiempo de espera en (1) es igual a la esperanza del coste de espera de cada consumidor. En este modelo asumiremos que todo el contenido tiene el mismo costo de demora por unidad de tiempo.

Por otro lado, en una red que prioriza el acceso con dos clases de prioridad, los costos de espera de los consumidores dependen de dichas clases. Basándose en resultados estándares de teoría de colas ${ }^{[17]}$, existen dos posibles esquemas de prioridad: el preferente y el no preferente.

Consideraremos en este caso que la priorización es del tipo "no preferente": un consumidor que solicita contenido designado como de primera clase de prioridad tiene el tiempo de espera determinado en (1), aplicado a este consumidor:

(2) $w_{1}=(\xi-\varphi 1)^{-1}$

Donde $\varphi_{1}$ es la cantidad total de tráfico desde consumidores que requieren contenido con la primera prioridad. Por el contrario, los consumidores que requieren contenido sin la primera prioridad, tendrán un tiempo de espera de 
$w_{2}=\xi *\left((\xi-\varphi)^{-1}\right) * w_{1}=$
(3) $=\left[\xi *\left((\xi-\varphi)^{-1}\right)\right] /\left[\xi *\left(\left(\xi-\varphi_{1}\right)^{-1}\right)\right]$

Basándonos en estos resultados estándar de la teoría de colas (Gross y Harris, 1998) podemos inferir que un consumidor tendrá un mayor costo de espera al solicitar el contenido no priorizado en lugar del priorizado:

(4) $w_{2}>w>w_{1}$ para $\xi>\varphi$

Esto se deduce al examinar la ratio relativa de $w_{2} a w_{1}$ que es $w_{2} / w_{1=} \xi *\left((\xi-\varphi)^{-1}\right)>1$, que es además una constante.

También la diferencia de calidad medida en los costos de espera se reduce a medida que aumenta la capacidad de la red, es decir:

$$
\frac{\partial}{\partial \zeta}(\chi)<0
$$

donde $\chi=\left(w_{2}-w_{1}\right)$

Esto se debe a que la reducción marginal del tiempo de espera para el tráfico priorizado debido a la expansión de la capacidad disminuye a medida que el nivel de capacidad se hace alto.

\section{4 Determinación del precio minorista del ISP}

En una red neutral, donde no hay posibilidad de realizar discriminación, los usuarios finales eligen a aquel proveedor de contenidos que les proporciona una mayor utilidad neta, considerando el costo de espera dado por (1). Conforme al modelo a la Hotelling, el consumidor marginal $\mathrm{x}^{*}$ esta indiferente entre los dos proveedores de contenido en la red neutral, y es definido por la siguiente igualdad de utilidades netas:

$$
v-(\xi-\varphi)^{-1}-t x^{*}-p=v-(\xi-\varphi)^{-1}-t-t x^{*}-p
$$

Donde es la utilidad bruta que le genera al consumidor su consumo de los servicios de contenidos, $(\xi-\varphi)^{-1}$ es el costo de espera definido en (1), -tx es el costo de transporte ${ }^{[18]}$ por la distancia hasta su localización y p es el precio minorista que paga al ISP por conectarse a internet ${ }^{[19]}$.

Los consumidores cuyas preferencias están representadas por $x<x^{*}$ eligen al $\mathrm{CAP}_{1}$, mientras que aquellos con $x>x^{*}$ eligen $\mathrm{CAP}_{2}$.

Si los dos proveedores de contenido están posicionados simétricamente, la provisión se divide por igual entre las dos empresas, y cada CAP atiende a la mitad del mercado, es 
decir, $\mathrm{x}^{*}=0,5$.

Consideramos que el parámetro de preferencias de cada consumidor $x$ es fijo, lo que implica que solo la restricción del consumidor medio es vinculante.

El problema de maximización de beneficios del ISP viene dado entonces, por:

$$
\underset{p}{\operatorname{Max}} \prod \text { ISP dado } v-(\xi-\varphi)^{-1}-t x^{*}-p \geq 0
$$

Donde la restricción es necesaria para asegurar la cobertura del mercado.

Podemos derivar entonces el precio de acceso minorista de equilibrio, el beneficio del ISP y de cada CAP:

(8) $\pi_{I S P}^{*}=p^{*}=v-(\xi-\varphi)^{-1}-\hat{t}$;

(9) $\pi_{C A P}^{*}=b_{i} \hat{\varphi} \quad$ parai $=1,2$

Con $\hat{t}=0,5 t \quad$ y $\quad \hat{\varphi}=0,5 \varphi$

\section{5 Determinación del precio minorista del ISP en una red no neutral}

Si se permite al ISP cobrar a los proveedores de contenido por un servicio de acceso de prioridad asegurada, los consumidores enfrentarán ahora diferentes tiempos de espera, dependiendo de su elección de CAP, tal como se expresa en (2) y (3).

Supongamos que el proveedor de contenido $\mathrm{CAP}_{1}$ obtiene la primera prioridad, por lo que su contenido tiene derecho a ser servido antes que el del $\mathrm{CAP}_{2}$.

Usaremos una tilde para tratar a variables asociadas al régimen de discriminación de velocidad: el consumidor marginal en $\widetilde{x}$ es indiferente entre el servicio con prioridad y el servicio básico, y se caracteriza por la siguiente igualdad:

$$
v-(\xi-\widetilde{x} \varphi)^{-1}-(t \tilde{x}-p)=v-\xi *(\xi-\phi)^{-1} *(\xi-\widetilde{x} \varphi)^{-1}-\hat{t}
$$

Donde $\hat{t}=t-t \tilde{x}-p$

Si consideramos lo expresado en las ecuaciones (4) y (7) podemos derivar en forma intuitiva que el CAP con la primera prioridad va a tener una participación de mercado mayor que el CAP que no tiene prioridad. Este resultado implica que:

$$
\widetilde{x} \geq x^{*}=0,5
$$


debido a la diferencia en los costos de espera, conforme lo expresado en (4).

En el escenario en que la discriminación es permitida, los beneficios del ISP incluyen un ingreso adicional al que cobra de los consumidores finales, y está representado por $\Omega$, el ingreso que obtiene el ISP por el servicio de prioridad cobrado al $\mathrm{CAP}_{1}$.

De esta forma, el problema de maximización del beneficio del ISP puede expresarse como:

$$
\text { Sujeto a } v-(\xi-\widetilde{x} \varphi)^{-1}-t^{\prime} \geq 0
$$

Donde $t^{\prime}=(t \tilde{x}-\widetilde{p})$.

Cuando ambos CAP compiten para adquirir el derecho a la prioridad, el ganador en la puja será aquel que tenga la mayor disponibilidad a pagar, esto es, será aquel que valore más la priorización.

Dado que la participación de mercado del CAP que adquiera la prioridad será $\widetilde{x}$ y la del que no lo adquiera será $(1-\widetilde{x})$, el máximo valor a pagar por la priorización será ${ }^{[20]}$ :

$$
b_{i}(2 \widetilde{x}-1) \phi
$$

Definamos además a $\Psi(0 \leq \Psi \leq 1)$ como el poder de negociación que mide la proporción de la renta que puede extraer el ISP del $\mathrm{CAP}_{1}$. En ese caso, el precio de la priorización será, conforme a (12):

$$
\begin{gathered}
\left.\Omega=(\Psi) b_{1}(2 \widetilde{x}-1) \varphi+(1-\Psi)\right) b_{2}(2 \widetilde{x}-1) \varphi \\
\Omega=\left(b_{2}+\Psi \Delta_{b}\right)(2 \widetilde{x}-1) \varphi
\end{gathered}
$$

Donde $\Delta_{b}=b_{1}-b_{2}(0)$

Cuanto mayor sea el poder de negociación que tenga el ISP, mayor será el precio que el CAP pague por la priorización.

Podemos entonces expresar el beneficio del ISP en el escenario de discriminación como:

$$
\begin{gathered}
\widetilde{\pi}_{\mathrm{ISP}}^{*}=\left(v-(\xi-\widetilde{x} \phi)^{-1}-t \widetilde{x}\right)+\left(b_{2}+\Psi \Delta_{b}\right)(2 \widetilde{x}-1) \phi \\
\widetilde{\pi}_{\text {ISP }}^{*}=\widetilde{p}+\Omega\left(14^{\prime}\right)
\end{gathered}
$$

Cuando el ISP asigna entonces la prioridad del acceso al $\mathrm{CAP}_{1}$ al precio (13), entonces el beneficio de cada CAP será: 


$$
\begin{gathered}
\tilde{\pi}_{\mathrm{CAP}_{1}}^{*}=\left(b_{1} \widetilde{x} \varphi\right)-\left(b_{2}+\Psi \Delta_{b}\right)(2 \widetilde{x}-1) \varphi \\
\tilde{\pi}_{\mathrm{CAP}_{21}}^{*}=b_{2}(1-\widetilde{x}) \varphi
\end{gathered}
$$

\section{6 Los efectos en los beneficios del ISP}

Podemos comparar entonces los beneficios del ISP en ambos escenarios: con regulación que obliga a la neutralidad (expresión 8), y con discriminación (expresión 14). Estos son:

$$
\begin{gathered}
\pi_{\text {ISP }}^{*}=p^{*}=v-(\zeta-\varphi)^{-1}-\hat{t} ;(8) \\
\tilde{\pi}_{\text {ISP }}^{*}=\left(v-(\zeta-\widetilde{x} \varphi)^{-1}-t \widetilde{x}\right)+\left(b_{2}+\Psi \Delta_{b}\right)(2 \widetilde{x}-1) \varphi
\end{gathered}
$$

El cargo por acceso a la red que paga el usuario en el escenario donde se permita la discriminación es menor que en el escenario de neutralidad, esto es $p^{*}>\widetilde{p}$ dado $w_{2}>w>$ $w_{1}$ conforme a (4) y a $\left(10^{\prime}\right)$.

Dado que en el escenario de discriminación el ISP cobra la una suma $\Omega$ a los CAP, encontramos el siguiente equilibrio potencial: el ISP optará por introducir un servicio de dos niveles si los ingresos procedentes por priorizar el tráfico superan el valor del menor ingreso obtenido del mercado minorista de acceso a Internet.

Este resultado amplía los resultados de Greenstein et al. (2016), que postulan que en mercados competitivos de acceso y de desarrollo de contenidos y en presencia de precios de dos vías, las cuotas de suscripción a consumidores finales disminuirán por la intensidad competitiva. En nuestro modelo, el ISP posee poder de mercado y aun así los pagos de los consumidores finales se reducen en el escenario de discriminación, dados los supuestos técnicos del modelo de tráfico considerado.

Eso redunda en beneficio de los usuarios finales, y es un aspecto que olvidado en el debate de políticas públicas.

Conforme a las especificaciones del modelo presentado podemos expresar que en un mercado donde no se observa el principio de neutralidad de red, el precio que el proveedor de servicios de Internet cobra a los usuarios finales se reduce respecto de una situación donde se observa el principio de neutralidad de red, independientemente de la competencia observada en el mercado de acceso a internet.

\section{Conclusiones y Comentarios}

Hemos ofrecido un análisis económico formal sobre los efectos de la reglamentación de la neutralidad de la red en beneficios de los ISP y los CAP, y sus implicaciones. 
Comparamos el equilibrio del mercado en el que se permite al ISP proporcionar un servicio de dos niveles vendiendo la vía rápida a un solo proveedor de contenido, con el equilibrio en el que no puede discriminar la velocidad de entrega del contenido.

En una red no neutral, donde se subasta la primera prioridad, ambos proveedores de contenido pueden participar en un juego del tipo dilema del prisionero para recibir la primera prioridad en la entrega de contenido y no estar en peor situación.

La decisión de los ISP de preferir el régimen discriminatorio a la red neutral depende del equilibrio entre el cargo de acceso a la red cobrado a los usuarios finales - que disminuye en el escenario de no neutralidad-y los ingresos provenientes de los CAP por el comercio de la primera prioridad.

Si se le da la oportunidad de cobrar al proveedor de contenido por la terminación, el proveedor de servicios de Internet está más dispuesto a disminuir la tasa de suscripción a los usuarios finales, precisamente porque se puede atraer a más usuarios finales para que se unan a la plataforma, lo que da lugar a más transacciones con los proveedores de contenido que también son rentables para el proveedor de servicios de Internet.

La neutralidad de red, en cambio, reduce los beneficios tanto de los proveedores de contenido como del ISP. No estarán interesados en generar tráfico adicional de ellos y esto genera ineficiencias.

\section{Referencias Bibliográficas}

- Armstrong, M. (2006). Competition in two-sided markets. The RAND journal of economics, 37(3), 668-691.

- Badasyan, N., \& Chakrabarti, S. (2003). Private peering among Internet backbone providers. Economics Working Paper Archive, Series on Industrial Organization, Washington University in St. Louis (WUSTL).

- Balachandran, K. R. (1972). Purchasing priorities in queues. Management Science, 18(5part-1), 319-326.

- Bourreau, M., Kourandi, F., \& Valletti, T. (2015). Net neutrality with competing internet platforms. The Journal of Industrial Economics, 63(1), 30-73.

- Bruno, N. (2019). Costos hundidos endógenos y competencia: aplicación al caso de telefonía local en EE. UU. Ensayos de Política Económica, 3(1), 37-57.

- Bruno, N. (2018). Introducción de competencia en los mercados de telecomunicaciones: Teoría y aplicación al caso de EE. UU. (No. 645). UCEMA, Serie Documentos de Trabajo.

- Bruno, N. (2005) UCEMA, T. A. Interconexión de redes entre dos operadores desregulados de acceso a Internet. 
- Caillaud, B., \& Jullien, B. (2003). Chicken \& egg: Competition among intermediation service providers. RAND journal of Economics, 309-328.

- Chambouleyron, A. (2002). ISP Interconnection and flat-rated internet pricing. Disponible en SSRN 316491.

- Choi, J. P., Jeon, D. S., \& Kim, B. C. (2014). Asymmetric neutrality regulation and innovation at the edges: fixed vs. mobile networks.

- Cheng, H. K., Bandyopadhyay, S., \& Guo, H. (2011). The debate on net neutrality: A policy perspective. Information systems research, 22(1), 60-82.

- Cisco, U. (2020). Cisco annual internet report (2018-2023) white paper. Online](accessed March 26, 2021)

https://www.cisco.com/c/en/us/solutions/collateral/executive-perspectives/annual-internet-re port/whitepaper-c11-741490.html.

- Coloma, G. (2005). Economía de la organización industrial (Vol. 197). Temas.

- Communication from the Commission. (2000). Unbundled access to the local loop:

Enabling the competitive provision of a full range of electronic communication services including broadband multimedia and high-speed Internet. C, 272, 55-66.

- Congress, U. S. (1996). Pub. L. No. 104-104, 110 Stat. 56. The Telecommunications Act of 1996.

- Crocioni, P. (2011). Net neutrality in Europe: Desperately seeking a market failure. Telecommunications Policy, 35(1), 1-11.

- Crémer, J., Rey, P., \& Tirole, J. (2000). Connectivity in the commercial Internet. The Journal of Industrial Economics, 48(4), 433-472.

- Cukier, K. (1999). The global Internet: a primer. Telegeography, 112-49.

- Dippon, C. M. \& Ware, H. (2010). Wholesale unbundling and intermodal competition. Telecommunications Policy, 34(1-2), 54-64.

- Edelson, N. M., \& Hilderbrand, D. K. (1975). Congestion tolls for Poisson queuing processes. Econometrica: Journal of the Econometric Society, 81-92.

- Economides, N. (1996). The economics of networks. International journal of industrial organization, 14(6), 673-699.

- Economides, P. N., Economides, N., \& Tåg, J. (2007). Net Neutrality on the Internet: A Two-sided Market Analysis. NET Institute Working Paper

- Economides, N. (2008). Net neutrality, non-discrimination and digital distribution of content through the internet. ISJLP, 4, 209. 
- Economides, N., \& Hermalin, B. E. (2012). The economics of network neutrality. The RAND Journal of Economics, 43(4), 602-629.

- Economides, N., \& Tåg, J. (2012). Network neutrality on the Internet: A two-sided market analysis. Information Economics and Policy, 24(2), 91-104.

- EUROPEIA, U. BEREC. Differentiation practices and related competition issues in the scope of Net Neutrality. Draft Report for public consultation.

https://eur-lex.europa.eu/legal-content/EN/TXT/PDF/?uri=CELEX:32015R2120\&from=en

- Federal Communications Commission. (2003). FCC Adopts New Rules for network unbundling obligations of incumbent local phone carriers. News Release: February, 20, 2003.

- Federal Communications Commission. (2005). Report and Order and Notice of Proposed Rulemaking, CC Docket No 02-33, 01-337, 95-20, 98-10, WC Docket Nos. 04-242, 05-271, Appropriate Framework for Broadband Access to the Internet over Wireline Facilities, (classifying wireline broadband Internet access service as an information service). News Release: September, 23, 2005.

- Gibbens, R., Mason, R., \& Steinberg, R. (2000). Internet service classes under competition. IEEE journal on selected areas in communications, 18(12), 2490-2498.

- Greenstein, S., Peitz, M., \& Valletti, T. (2016). Net neutrality: A fast lane to understanding the trade-offs. Journal of Economic Perspectives, 30(2), 127-50.

- Gross, D., \& Harris, C. M. (1998). Fundamentals of Queueing Theory, 3rd eddition. John Weiley and Sons.

- Guo, H., \& Easley, R. F. (2016). Network neutrality versus paid prioritization: Analyzing the impact on content innovation. Production and Operations Management, 25(7), 1261-1273.

- Hahn, R. W., \& Wallsten, S. (2006). The Economics of Net Neutrality (April 2006). AEIBrookings Joint Center Working Paper No. RP06-13, online unter: http://ssrn. com/abstract, 943757.

- Hausman, J. A., \& Sidak, J. G. (2005). Did mandatory unbundling achieve its purpose? Empirical evidence from five countries. Journal of Competition Law and Economics, 1(1), 173-245.

Hazlett, T. W., \& Caliskan, A. (2008). Natural experiments in US broadband regulation. Review of Network Economics, 7(4).

- Hermalin, B. E., \& Katz, M. L. (2004). Sender or Receiver: Who should pay to exchange an electronic message?. RAND Journal of Economics, 423-448. 
- Hermalin, B. E., \& Katz, M. L. (2007). The economics of product-line restrictions with an application to the network neutrality debate. Information Economics and Policy, 19(2), 215-248.

- International Energy Agency (25 de marzo 2021) Global trends in internet traffic, data centre workloads and data centre energy use, 2010-2019.

https://www.iea.org/data-and-statistics/charts/global-trends-in-internet-traffic-data-centre-w orkloads-and-data-centre-energy-use-2010-2019

- Jeon, D. S., Laffont, J. J., \& Tirole, J. (2004). On the" receiver-pays" principle. RAND Journal of Economics, 85-110.

Jullien, B., \& Sand-Zantman, W. (2018). Internet regulation, two-sided pricing, and sponsored data. International Journal of Industrial Organization, 58, 31-62.

- Katz, M. L., \& Shapiro, C. (1985). Network externalities, competition, and compatibility. The American economic review, 75(3), 424-440.

- Krämer, J., \& Wiewiorra, L. (2012). Network neutrality and congestion sensitive content providers: Implications for content variety, broadband investment, and regulation. Information Systems Research, 23(4), 1303-1321.

- Kocsis, V., \& de Bijl, P. W. (2007). Network neutrality and the nature of competition between network operators. International Economics and Economic Policy, 4(2), 159-184.

- Laffont, J. J., Rey, P., \& Tirole, J. (1997). Network Competition: Part I and II. Cahier de IIDEI, (65).

- Laffont, J. J., Rey, P., \& Tirole, J. (1998). Network competition: I. Overview and nondiscriminatory pricing. The RAND Journal of Economics, 1-37.

- Laffont, J. J., Rey, P., \& Tirole, J. (1998). Network competition: II. Price discrimination. The RAND Journal of Economics, 38-56.

- Mendelson, H. (1985). Pricing computer services: Queueing effects. Communications of the ACM, 28(3), 312-321.

- Mendelson, H., \& Whang, S. (1990). Optimal incentive-compatible priority pricing for the M/M/1 queue. Operations research, 38(5), 870-883.

- Miller, C. C., \& Stone, B. (2010). App Makers Worry as Data Plans Are Capped. The New York Times, 6

- Naor, P. (1969). The regulation of queue size by levying tolls. Econometrica: journal of the Econometric Society, 15-24.

- Peitz, M., \& Schuett, F. (2016). Net neutrality and inflation of traffic. International Journal of Industrial Organization, 46, 16-62. 
- Reggiani, C., \& Valletti, T. (2016). Net neutrality and innovation at the core and at the edge. International Journal of Industrial Organization, 45, 16-27.

- Rochet, J. C., \& Tirole, J. (2003). Platform competition in two-sided markets. Journal of the european economic association, 1(4), 990-1029.

- Rochet, J. C., \& Tirole, J. (2006). Two-sided markets: a progress report. The RAND journal of economics, 37(3), 645-667.

- Tarziján, J., \& Paredes, R. (2001). Organización industrial para la estrategia empresarial. Pearson Education

- Weiss, M. B., \& Shin, S. J. (2004). Internet interconnection economic model and its analysis: Peering and settlement. Netnomics, 6(1), 43-57.

- Weisman, D. L., \& Kulick, R. B. (2010). Price discrimination, two-sided markets, and net neutrality regulation. Tul. J. Tech. \& Intell. Prop., 13, 81.

- Svensson, P. (19 de octubre de 2007) Comcast Blocks Some Internet Traffic. NBC News. https://www.nbcnews.com/id/wbna21376597

- Somogyi, R. (2016). The economics of zero-rating and net neutrality. CORE Discussion Papers 2016047, Université catholique de Louvain. Center for Operations Research and Econometrics (CORE).

\section{Acerca del Autor}

Néstor Bruno: economista regulatorio experto en Telecom Argentina S.A, investigador del Instituto de Economía de la Universidad Argentina de la Empresa, profesor titular en la misma, y Doctor en Economía por UCEMA.

\section{Notas}

Utilizaremos indistintamente el concepto de "no neutralidad" y "discriminación"

$\uparrow 1$ toda vez que la discriminación entre distintos usuarios implica una práctica no neutral. 
El 19 de octubre de 2007, The Associated Press (AP) informó que Comcast, el mayor operador de televisión por cable de Estados Unidos y segundo mayor proveedor de Internet, había interferido con el acceso de los usuarios a sitios de intercambio de archivos como BitTorrent. Esta práctica es un ejemplo de discriminación en el que los ISP tenían la intención de ralentizar algunas formas de tráfico, al tiempo que daba prioridad a otras.

Una definición generalmente aceptada de facilidad esencial es la que provee la OECD (2006) "la infraestructura esencial significa que prestar un servicio es sustancialmente más difícil sin acceso a esta infraestructura, y que el propietario monopolista de esta infraestructura encontraría rentable imponer al menos un aumento de precio reducido pero significativo y no transitorio sobre el nivel competitivo para acceder a esta infraestructura". La doctrina de las facilidades fsenciales es muy antigua y se originó en Estados Unidos en 1912 con un caso sobre los servicios de ferrocarriles: "United States vs. Terminal Railroad Association of Saint Louis, 1912".

El paradigma detrás de ese esquema fue la creencia de que la reducción de barreas de entrada implicaría una afluencia de competidores a la industria, los que al no tener que hundir costos en la construcción de nuevas redes podrían ocuparse de generar competencia por una cuota de mercado y solo cuando lo hubieran obtenido construirían las mismas, aumentando la capacidad total ofrecida y cambiando la configuración del mismo. La concentración de la industria, sin embargo, se mantuvo elevada a pesar de las medidas que pretendieron introducir competencia utilizando la red del operador ya establecido debido a la existencia de costos hundidos endógenos en la industria.

Para un análisis sobre el resultado de este tipo de políticas de apertura a la competencia, denominada genéricamente doctrina de las facilidades esenciales, ver Dippon y Harold (2010), Hausman y Sidak (2004), Hazlett y Caliskan (2008) y más recientemente Bruno, (2018).

Una serie de acuerdos y contratos permiten a los consumidores acceder al contenido disponible en todo el mundo. En el núcleo de Internet (core de Internet) estos toman la forma de acuerdos de peering mientras que, si el tamaño o cobertura de los ISP es desigual y el tráfico entre ellos es desequilibrado, implicara contratos de tránsito y pagos entre las partes.

Son prestadores del servicio que se valen del arrendamiento de infraestructura al operador establecido. Este debe ofrecer usualmente acceso a sus elementos de red como remedio regulatorio a su posición de dominio en el mercado relevante. 
Dado que el tráfico enviado y recibido es similar, no se originan pagos recíprocos. La similitud de tráficos implica la expresión de pares o peering.

Webhosting es el servicio que provee a los usuarios de Internet un sistema para poder almacenar información, imágenes, video, o cualquier contenido accesible vía web.

Desde el punto de vista de los reguladores y organismos de defensa de la competencia, si bien no rechazan esta práctica, se analizan las ofertas zero rate en

$\uparrow 10$ un modo caso por caso y se considera que si el zero rate se adjudica solo en una aplicación específica esto socavará los derechos de los usuarios finales o puede conducir a circunstancias en las que la elección de los usuarios finales se reduzca

Aquí se avizora otros de los temas regulatorios actuales: el incentivo de los ISP (y $\uparrow 11$ operadores de red) a invertir en redes de mayor capacidad, cuando el precio a cobrar no está relacionado con el uso de cada participante.

Miller \& Stone (2010) ejemplifican esta situación en el mercado móvil en EE. UU.: dado que el regulador estadounidense (FCC) no aceptaba cargos de ISP a los CAP, los operadores como AT\&T introdujeron límites de uso de Internet para administrar la congestión. Es la forma en que los ISP pueden elegir para racionar la demanda, actuando del lado de los consumidores.

Si bien puede ser relativamente fácil identificar qué servicios generan intenso tráfico en horas pico y que también sean menos sensibles a los retrasos, diseñar tarifas minoristas que logren el mismo efecto para estos patrones de tráfico puede ser más complejo, Crocioni (op.cit.).

$\uparrow 14$ Corresponde a la traducción de la expresión inglesa hazard rate.

Economides et al. (2007) y Cheng et al. (2009) modelan los ingresos de los CAP $\uparrow 15$ considerando que ofrecen sus servicios sin ningún cargo directo a los consumidores y solo obtienen ingresos a través de la publicidad.

El beneficio del CAP correspondiente se mide como $b_{i \varphi} \sigma_{i}$ donde $\sigma_{i}$ representa la cuota de mercado para el proveedor de contenido i. 
Véase Gross y Harris (1998) y sus referencias para más información sobre la teoría $\uparrow 17$ de colas y para la derivación detallada de los costos de espera en diferentes tipos de redes.

Conforme a Coloma (2005) el costo de transporte se interpreta literalmente en casos en los cuales la diferenciación es propiamente geográfica, mientras que, si el espacio relevante es el de otra característica, " $t$ " pasa a ser equivalente al costo en términos de utilidad de un consumidor que preferiría una determinada variedad, pero termina comprando otra que se encuentra a una cierta distancia de su opción teóricamente preferida.

En este modelo asumimos que no hay pagos directos entre el CAP y el consumidor final, lo que simplifica el análisis.

Economides (2008) analiza varias consecuencias de la desviación de la regulación \20 de neutralidad de la red basada en la subasta de priorización a través de la cual solo un grupo de proveedores de contenidos tiene derecho a la vía rápida. 